\title{
Monitoring polypharmacy in healthcare systems through a multi-setting survey: should we put more attention on long term care facilities?
}

\author{
Luca Arnoldo, ${ }^{1}$ Giovanni Cattani, ${ }^{1}$ Piergiorgio Cojutti, ${ }^{2}$ Federico Pea, ${ }^{2}$ Silvio Brusaferro' \\ ${ }^{1}$ Department of Medical and Biological Sciences, University of Udine; ${ }^{2}$ Institute of Clinical \\ Pharmacology, Complex Local Health Unit, Udine, Italy
}

\begin{abstract}
Significance for public health
We believe that our research is appropriate for your journal because concerns a very important issue for healthcare systems: the management of drugs in elderly people, in particular our study evaluated the topic of polypharmacy and potentially inappropriate prescriptions in a whole regional healthcare system, considering the three main care settings: acute hospitals, long term care facilities and general practitioners' clinics. We also analysed the problem included all the medications that patients assumed, not only ones that were reimbursed by healthcare system. Considering these premises this study permitted to have a wide point of view for setting tailored policies and it underlines the importance of creating a system that starts from real data and in this way will allows to compare and measure improvement actions.
\end{abstract}

\section{Abstract}

Background. Polypharmacy is a main issue of patient safety in all healthcare settings (i.e. increase adverse drug reactions and incidence of drug-drug interactions, etc.). The main object of the study was to evaluate the prevalence of polypharmacy and the appropriateness of drugs prescriptions in the regional health system (RHS) of Friuli Venezia-Giulia Region, Italy.

Design and methods. We carried out a point prevalence study in May 2014 ; 1582 patients $\geq 65$ years were included from: 14 acute hospitals, 46 Long Term Care Facilities (LTCFs) and 42 general practitioners' (GPs) clinics. Data analysis included the evaluation of potentially inappropriate prescriptions (PIPs) taking Beers criteria as a reference.

Results. Patients in therapy with 10 drugs or more were $13.5 \%: 15.2 \%$ in hospitals, $9.7 \%$ in GPs' clinics and $15.6 \%$ in LTCFs. According to Beers criteria we identified 1152 PIPs that involved globally almost half of patients (46.0\%): 41.9\% in hospitals, 59.6\% in LTCFs and 37.0\% in GP's clinics. The $53.9 \%$ of patients received at least one mainly kidney excreted drug; for these patients the evaluation of serum creatinine was overall present in the $87.7 \%$ (747/852): $96.4 \%$ in hospital ones, $87.5 \%$ in GPs' clinics and $77.8 \%$ in LTCFs. LTCFs residents were significantly $(\mathrm{P}<0.05)$ more exposed to PIPs and less monitored for the renal function.

Conclusions. A reliable estimation of the phenomenon in all the main healthcare settings is a necessary prerequisite to set tailored policies for facing polypharmacy within a RHS; the results showed the necessity to put a special attention on LTCFs.

\section{Introduction}

Polypharmacy represents a main patient safety issue in all healthcare settings worldwide. ${ }^{1}$ Although it can potentially concern people of any ages, the elderly are most frequently involved: the availability of a greater number of drugs, the increase of life expectancy and a higher prevalence of chronic diseases represent the main reasons for a higher incidence in this population. ${ }^{2}$

Many definitions of polypharmacy, both qualitative and quantitative, are currently used: medication does not match the diagnosis, excessive use of medication, duplication of medication, medication prescribed to treat the side effect of another medication, ${ }^{3}$ but the simultaneous intake of many medications (generally five or more) is the one more frequently adopted..$^{4-6}$

The prevalence of polypharmacy in Europe ranges from to $33.8 \%$ to $73.3 \%$ depending on definition reported; 7,8 official data regarding the prevalence of polypharmacy in Italian elderly (approximately 12 million of people $\geq 65$ years old) report that 6 million people were treated with 5-9 drugs and 1.3 million with 10 drugs or more. ${ }^{9}$ These data potentially underestimate the phenomenon because they did not include medications not reimbursed by the national pharmaceutical formulary, such as benzodiazepines and some of non-steroidal antiinflammatory drugs (NSAIDs).

Possible consequences of polypharmacy include: increase of adverse drug reactions (ADR), incidence of drug-drug interactions, decrease in patient compliance to treatment, increase of risk of falling, inability and cost increase for healthcare system, ${ }^{10-13}$ due to an higher rate of hospitalisation, morbidity and mortality.

Although general data are available on polypharmacy, only few ones are available about its presence in the main healthcare settings: primary care, LTCFs and hospitals. A detailed picture at healthcare system level is, in fact, a first step for facing polypharmacy. This paper reports the results of a multi-setting survey on polypharmacy conducted in a regional healthcare system with the aim to identify strengths and weaknesses of the different settings as premise for the definition of tailored actions.

\section{Design and Methods}

The study was carried out in the regional healthcare system (RHS) of Friuli Venezia Giulia (FVG), Italy, in March 2014 and explored the three main settings that provide care to elderly: a) all the fourteen public and private acute hospitals of the RHS, b) 46 (out of 90) long term care facilities (LTCFs) (22 nursing homes and 24 long term chronic care facilities) and c) 42 volunteer general practitioners (GPs) representing all the 22 local health districts of RHS.

Eligible people were all the 65 years old or more who, at the moment of the survey, were taking at least one chronic or acute drug. Oncologic drugs and topical medications (i.e. eye-drops and ointments) were excluded as well as homeopathic and natural ones.

The sample was recruited applying a specific approach in each setting: a) for hospitals all the eligible patients discharged from internal 
medicine, geriatrics, cardiology and neurology wards in seven consecutive working days; b) for LTCFs, that included two types of facilities differing by intensity of care and length of stay, we carried out the survey in a single day and included the $30 \%$ (randomly selected) of eligible residents (from an overall sample of 739 residents) in long-term chronic care facilities and the $10 \%$ in the nursing homes (from an overall sample of 2410 residents). The selected survey index day for LTCFs was the first one of the survey in hospitals and GPs, this choice was made to avoid the bias that residents in LTCFs could be re-evaluated during a following admission in hospital; c) for GPs the sample was composed by the first two eligible people who accessed to the GPs' clinics each day for seven consecutive working days. For each patient we anonymously collected: age, gender, weight, most recent blood values of creatinine (within a year), presence of specific chronic diseases, prescribed medication name, the therapy dose and length. All prescribed medications were collected, also not RHS reimbursed and pro re nata (PRN) therapy. The survey methodology was preliminary tested in two wards (geriatrics and cardiology) of two hospitals and in three LTCFs. Moreover a two hours course to train data collectors was organised by the coordination team and included all the 60 professionals involved in the survey.

Data were collected by reviewing medical records for hospitalised patients and LTCFs residents; for outpatients all necessary information was provided by the GPs through their electronic databases.

Medications were classified following the Anatomical Therapeutic Chemical (ATC). On the basis of available literature on topic, ${ }^{4-6}$ exposition to medications was stratified into three classes: from 1 to 4 , from 5 to 9 (polypharmacy) and 10 or more (hyper-polypharmacy).

We also analysed the presence of potentially inappropriate prescription (PIP) defined using the 2012 Beers Criteria. ${ }^{14}$

The study protocol was approved by the Regional Ethical Committee in March 2014.

Categorical variables were compared using Shapiro-Wilk test for evaluating normality, Pearson test for linear correlation, Kruskal-Wallis test for non-parametric data and logistic regression model. We accepted a $\mathrm{P}<0.05$ as a significant value.

All data analysis was performed using IBM SPSS Statistics 20.

\section{Results}

The survey involved overall 1582 patients; their characteristics, stratified by setting, are summarised in Table 1 .
The mean \pm SD age of the study population was $80.5 \pm 8.4$, with a significant variability $(\mathrm{P}<0.05)$ by setting: hospitals $80.8 \pm 8.1$, GPs $76.7 \pm 7.8$ and LTCFs $84.1 \pm 7.8$. Females were the majority $54.7 \%$ (323/591) and $74.9 \%$ (420/561) respectively in the 75-84 and in the 85 or more classes of age, while males were the majority in the 65-74 class $55.6 \%$ (239/430). Almost all patients (96.4\%) suffered at least of one disease with a mean \pm SD of $3.4 \pm 1.5$ diseases for patients (range from 0 to 12 ): hospitals $3.2 \pm 1.8$, GPs $3.1 \pm 2.0$ and LTCFs $3.2 \pm 1.7$. Hypertension resulted the most common reported chronic disease $60.2 \%$ (953/1582), followed by arrhythmic disorders $27.7 \%$ (438/1582), heart diseases 25.9\% (410/1582), diabetes mellitus 25.8\% (408/1582), arthropathy 25.5\% (403/1582) and cerebro-vascular artery disease $25.3 \%$ (400/1582). The most common associations resulted: hypertension and diabetes mellitus $17.9 \%$ (283/1582), hypertension and arrhythmic disorders 17.6\% (278/1582) and hypertension and heart diseases $17.2 \%(272 / 1582)$.

The mean \pm SD number of prescribed drugs for each patient resulted $6.1 \pm 3.0$.The mean number was significantly lower $(\mathrm{P}<0.05)$ for GPs' outpatients $(5.5 \pm 3.0)$ than for hospitalised patients $(6.4 \pm 2.9)$ and LTCFs residents $(6.3 \pm 2.9)$. There was a moderate significant linear correlation $(\mathrm{P}<0.05)$ between the numbers of diseases and number of drugs assumed (Pearson's test 0.37 ).

Cardiovascular system (36.3\%, 3485 out of 9599), alimentary tract and metabolism $(20.0 \%, 1923$ out of 9599) and blood and blood forming organs $(14.9 \%, 1427$ out of 9599$)$ were the three ATC drugs categories most frequently prescribed; Table 2 specifies the five most common ATC classes for each setting.

We observed that $53.9 \%(852 / 1582)$ of patients received at least one mainly kidney excreted drug (ramipril, metformin, allopurinol, digoxin, enalapril, nebivolol, levofloxacin, atenolol, levetiracetam and pregabalin); for these patients the evaluation of serum creatinine (necessary for estimating renal function) was overall present in the $87.7 \%$ (747/852), with significant difference in the three settings $(\mathrm{P}<0.05)$ : $96.4 \%$ (295/306) in hospital ones, $87.5 \%$ (245/280) in GPs' clinics and $77.8 \%(207 / 266)$ in LTCFs.

According to Beers criteria we found 1152 PIPs that involved almost half of patients (46.0\%, 728 out of 1582): 41.9\% (219/528) in hospitals, $59.6 \%$ (314/527) in LTCFs and 37.0\% (195/527) in GP's clinics; Table 3 shows the univariate analysis and the logistic regression model for the presence of at least one PIP.

All 1582 patients were included in the logistic regression model and we evaluated the parameters that resulted statistically significant $(\mathrm{P}<0.05)$ in the univariate analysis. Results highlight that the risk of

Table 1. Studied population characteristics by explored setting.

\begin{tabular}{|c|c|c|c|c|}
\hline & $\begin{array}{c}\text { Hospitals (n. 528) } \\
\text { n. (\%) }\end{array}$ & $\begin{array}{l}\text { GPs (n. 527) } \\
\text { n. }(\%)\end{array}$ & $\begin{array}{c}\text { LTCFs (n. 527) } \\
\text { n. (\%) }\end{array}$ & $\begin{array}{c}\text { Overall (n. 1582) } \\
\text { n. (\%) }\end{array}$ \\
\hline Females & $261(49.3)$ & $302(57.3)$ & $371(70.4)$ & $934(59.0)$ \\
\hline $\begin{array}{c}\text { Age classes } \\
\quad 65-74 \\
75-84 \\
\geq 85 \\
\end{array}$ & $\begin{array}{l}128(24.2) \\
205(38.8) \\
195(36.9)\end{array}$ & $\begin{array}{c}233(44.2) \\
203(38.5) \\
91(17.3)\end{array}$ & $\begin{array}{c}69(13.1) \\
183(34.7) \\
275(52.2)\end{array}$ & $\begin{array}{l}430(27.2) \\
591(37.4) \\
561(35.5)\end{array}$ \\
\hline $\begin{array}{l}\text { N. of prescribed drugs } \\
\begin{array}{l}1-4 \\
5-9 \\
\geq 10\end{array}\end{array}$ & $\begin{array}{l}139(26.3) \\
309(58.5) \\
80(15.2)\end{array}$ & $\begin{array}{c}223(42.3) \\
253(48.0) \\
51(9.7)\end{array}$ & $\begin{array}{c}157(29.8) \\
288(54.6) \\
82(15.6)\end{array}$ & $\begin{array}{l}519(32.8) \\
850(53.7) \\
213(13.5)\end{array}$ \\
\hline $\begin{array}{c}\text { Number of diseases } \\
0-1 \\
2-4 \\
\geq 5\end{array}$ & $\begin{array}{l}80(15.2) \\
317(60.0) \\
131(24.8)\end{array}$ & $\begin{array}{c}51(9.7) \\
300(56.9) \\
176(33.4)\end{array}$ & $\begin{array}{l}83(15.7) \\
342(64.9) \\
102(19.4)\end{array}$ & $\begin{array}{l}214(13.5) \\
959(60.6) \\
409(25.9)\end{array}$ \\
\hline
\end{tabular}


PIP increases with the number of assumed drugs (OR 2.77 and 5.60 respectively for 5-9 class and $\geq 10$ class compare to 1-4); also the setting has an important influence, in fact hospital and GPs' patients have a significantly lower risk (OR 0.46 for both) compared to elderly people in LTCFs.

\section{Discussion and Conclusions}

The study was carried out to obtain an overall picture of medications use in elderly people for the different RHS settings; sample heterogene- ity was a necessary requisite to understand the problem in all its complexity; the three involved settings represent the main care providers for elderly in the RHS so a specific knowledge of their situation was considered an essential prerequisite for the definition and implementation of actions and setting policies.

In this perspective, the prevalence survey was the methodology for reaching our goal; it allowed us both to recruit many patients in a limited time and to collect all the prescribed drugs (included RHS not reimbursed and PRN ones), two requisites necessary for a thorough picture as close as possible to reality. Furthermore this approach was able to overcome the existing differences in the way clinical data are reported, collected and stored in the 3 different settings and within

Table 2. The five most common prescribed drug classes stratified by setting.

\begin{tabular}{|c|c|c|c|c|c|c|c|c|}
\hline \multirow{2}{*}{$\begin{array}{l}\text { Hospitals (n. 3386) } \\
\text { Drug class }\end{array}$} & \multirow[b]{2}{*}{ n. } & \multirow[b]{2}{*}{$\%$} & \multicolumn{3}{|c|}{ General practitioners (n. 2872) } & \multicolumn{3}{|l|}{ LTCFs (n. 3341) } \\
\hline & & & Drug class & n. & $\%$ & Drug class & n. & $\%$ \\
\hline B01 Antithrombotic agents & 481 & 14.2 & $\begin{array}{l}\text { C09 Agents acting on } \\
\text { the renin-angiotensin system }\end{array}$ & 374 & 13.0 & $\begin{array}{l}\text { B01 Antithrombotic } \\
\text { agents }\end{array}$ & 425 & 12.7 \\
\hline A02 Drugs for acid related disorders & 379 & 11.2 & B01 Antithrombotic agents & 330 & 11.5 & $\begin{array}{l}\text { A02 Drugs for acid } \\
\text { related disorders }\end{array}$ & 380 & 11.4 \\
\hline C03 Diuretics & 315 & 9.3 & C10 Lipid modifying agents & 222 & 7.7 & N05 Psycholeptics & 284 & 8.5 \\
\hline $\begin{array}{l}\text { C09 Agents acting on the } \\
\text { renin-angiotensin system }\end{array}$ & 267 & 7.9 & $\begin{array}{l}\text { A02 Drugs for acid related } \\
\text { disorders }\end{array}$ & 214 & 7.5 & C03 Diuretics & 251 & 7.5 \\
\hline C07 Beta blocking agents & 243 & 7.2 & C07 Beta blocking agents & 191 & 6.7 & $\begin{array}{l}\text { C09 Agents acting } \\
\text { on the renin-angiotensin } \\
\text { system }\end{array}$ & 211 & 6.3 \\
\hline
\end{tabular}

Table 3. Logistic regression model and univariate analysis of the presence of at least one potentially inappropriate medication (PIP) for Beers criteria.

\begin{tabular}{|c|c|c|c|c|c|c|c|c|}
\hline & Presence & f at least one PIP & & iate a & & & c regi & ion \\
\hline & HIs Expuseu & & Sig. & OR & $95 \% \mathrm{CI}$ & Sig. & OR & $95 \% \mathrm{CI}$ \\
\hline $\begin{array}{l}\text { Gender } \\
\quad \text { Female } \\
\text { Male }\end{array}$ & $\begin{array}{l}452 \\
276\end{array}$ & $\begin{array}{l}482 \\
372\end{array}$ & 0.02 & 1.26 & $1.03-1.55$ & 0.08 & 1.22 & $0.97-1.53$ \\
\hline $\begin{array}{r}\text { Age class } \\
75-84 \\
65-74\end{array}$ & $\begin{array}{l}284 \\
163\end{array}$ & $\begin{array}{l}307 \\
267\end{array}$ & 0.00 & 1.52 & $1.18-1.95$ & 0.26 & 1.19 & $0.90-1.56$ \\
\hline $\begin{array}{c}\text { Age class } \\
\geq 85 \\
65-74\end{array}$ & $\begin{array}{l}281 \\
163\end{array}$ & $\sqrt{n+3}$ & 0.00 & 1.64 & $1.27-2.12$ & 0.47 & 1.12 & $0.83-1.50$ \\
\hline $\begin{array}{l}\text { Setting } \\
\text { Hospitals } \\
\text { LTCFs }\end{array}$ & $\begin{array}{l}219 \\
314\end{array}$ & $\begin{array}{l}309 \\
213\end{array}$ & 0.00 & 0.48 & $0.38-0.62$ & 0.00 & 0.46 & $0.35-0.60$ \\
\hline $\begin{array}{l}\text { Setting } \\
\text { GPs } \\
\text { LTCFs }\end{array}$ & $\begin{array}{l}195 \\
314\end{array}$ & $\begin{array}{l}332 \\
213\end{array}$ & 0.00 & 0.40 & $0.31-0.51$ & 0.00 & 0.46 & $0.35-0.60$ \\
\hline $\begin{array}{c}\text { Diseases } \\
2-4 \\
0-1\end{array}$ & $\begin{array}{c}448 \\
77\end{array}$ & $\begin{array}{l}511 \\
137 \\
\end{array}$ & 0.04 & 1.56 & $1.15-2.12$ & 0.16 & 1.26 & $0.91-1.76$ \\
\hline $\begin{array}{c}\text { Diseases } \\
\geq 5 \\
0-1\end{array}$ & $\begin{array}{c}203 \\
77\end{array}$ & $\begin{array}{l}206 \\
137\end{array}$ & 0.01 & 1.75 & $1.25-2.46$ & 0.33 & 1.21 & $0.83-1.76$ \\
\hline $\begin{array}{l}\text { Prescribed drugs } \\
\quad 5-9 \\
1-4\end{array}$ & $\begin{array}{l}439 \\
143\end{array}$ & $\begin{array}{l}411 \\
376\end{array}$ & 0.00 & 2.81 & $2.22-3.55$ & 0.00 & 2.77 & $2.16-3.54$ \\
\hline $\begin{array}{l}\text { Prescribed drugs } \\
\quad \geq 10 \\
1-4\end{array}$ & $\begin{array}{l}146 \\
143\end{array}$ & $\begin{array}{c}67 \\
376\end{array}$ & 0.00 & 5.73 & $4.05-8.11$ & 0.00 & 5.60 & $3.87-8.12$ \\
\hline
\end{tabular}


each setting. The sample recruitment was set with different methodologies in the three settings adopting a tailored strategy for the different characteristics of patients' stay: acute ones, residents and outpatients.

The overall prevalence of polypharmacy (67.2\%) was quite similar to the results (61.0\%) of the national prevalence study conducted by Geriatric Working Group of Italian Agency of Drugs (AIFA) in 2011;15 the gap between the two scenarios could be explained by the absence of the not reimbursed drugs by national pharmaceutical formulary in the AIFA one. Reliability of our data can also be confirmed by the increase in number of chronic diseases correlated to the number of prescribed drugs, as highlighted in literature. ${ }^{16,17}$

Taking Beers criteria as reference, ${ }^{14}$ the survey revealed that approximately half of patients (46.0\%) had at least one PIP in their therapy, substantially similar to the results of other available experiences which analysed only single settings. ${ }^{18,19}$ In our case, the analysis by setting allowed a comparison and LTCFs emerged as most critical: almost 6 patients out of 10 presented a PIP. This result was significantly higher $(\mathrm{P}<0.05)$ compared to the other settings, also considering that the majority of LTCFs residents were followed by their own GPs and that in LTCFs the prevalence of 5 or more diseases was lower compared to GPs' outpatients.

Furthermore through this approach we found that one of the main causes of PIPs occurrence was the significant $(\mathrm{P}<0.05)$ higher use of psycholeptic drugs in LTCFs compared to the other two settings.

Also data regarding the evaluation of renal function for patients who were taking at least one drug with renal excretion confirmed that LTCFs had a lower level of compliance compared to the other two settings; this is an important issue for patient safety because the knowledge of renal function is necessary to adapt drugs doses and to prevent accumulation complications.

The study identified a problem that must be necessarily considered primary for patient safety in a context of regional risk management approach, the stewardship of long term care providers for elderly also considering the emerging change in population demography.

A strength of the proposed approach is the ability to provide a full view of the state of the art about drugs prescription quality in a RHS; it could potentially be a starting point for policies aimed to improve patient safety and safer drug use: based on these results it is possible to communicate the state of the art to all interested stakeholders and to set tailored improvement programmes. Although there are many papers describing polypharmacy in elderly population almost all of them explored only one care setting and did not included systematically all prescribed drugs.

Regional plans should consider educational programmes for prescribers, continuous data flow on drugs use to all healthcare practitioners, sharing of good practices about drugs management (i.e. the periodic re-evaluation of patient's therapy, the careful check of possible drug-drug interaction and of potentially inappropriate medications, etc.) and patient literacy on drugs use, ${ }^{20,21}$ further medication reconciliation should be systematically applied in all the healthcare settings and with particular attention in LTCFs, as they showed the greatest polypharmacy concern.

The routinely evaluation of renal function in elderly patients taking mainly kidney excreted drugs is one of the most important, and possibly less expensive, improvement initiatives suggested by our findings and it will be a priority for ensuring the right dose of drugs in this way preventing accumulation.

These data as well as their periodical repetition could be very helpful both in making clear different aspects of the problem locally and monitoring possible progresses.

The proposed approach had also some limitations and particularly, first one was that GPs' recruitment for outpatients was only possible on voluntary basis and it consequently could had selected the more moti- vated ones; another one was that GPs' outpatients, in spite of presence of higher prevalence of diseases, were usually more independent for own care management compared to LTCFs ones. Furthermore the adoption of only the Beers criteria could have underestimated PIPs rate if compared with studies that utilised also Screening Tool of Older Persons' Potentially inappropriate Prescriptions (STOPP), ${ }^{22,23}$ but for our aim it was more important adopting an unique standardised tool that allows us to compare the three settings.

In conclusion, the availability of reliable estimation of the phenomenon in all the main healthcare settings of RHS is a necessary pre-requisite for setting up effective policies aimed to control polypharmacy. In our context LTCFs seemed to need special attention compared to hospitals and GPs' clinics. The definition of policies and programmes at health system level needs a periodical monitoring of polypharmacy in the main settings of the system (primary care, LTCFs and hospitals) and setting tailored interventions for improving and promoting medication reconciliation process and drug management.

Correspondence: Luca Arnoldo, Department of Medical and Biological Sciences, University of Udine, via Colugna 50, 33100 Udine, Italy.

Tel.: +39.043.2559207.

E-mail: luca.arnoldo@uniud.it

Key words: Drugs; Elderly; Health policy; Polypharmacy, LCTFs.

Contributions: the authors contributed equally.

Conflict of interest: the authors declare no potential conflict of interest.

Received for publication: 29 September 2016.

Accepted for publication: 29 0ctober 2016.

(C) Copyright L. Arnoldo et al., 2016

Licensee PAGEPress, Italy

Journal of Public Health Research 2016;5:745

doi:10.4081/jphr.2016.745

This work is licensed under a Creative Commons Attribution NonCommercial 4.0 License (CC BY-NC 4.0).

\section{References}

1. Sharifi H, Hasanloei AH, Mahmoudi. Polypharmacy-induced drugdrug interactions; threats to patient safety. Drug Res 2014;64:6337 .

2. Patterson SM, Hughes C, Kerse N, et al. Interventions to improve the appropriate use of polypharmacy for older people. Cochrane Database Syst Rev. 2012;5:CD008165.

3. Bushardt RL, Massey EB, Simpson TW, et al. Polypharmacy: misleading, but manageable. Clin Interv Aging 2008;3:383-9.

4. Jörgensen T, Johansson S, Kennerfalk A, et al. Prescription drug use, diagnoses, and healthcare utilization among the elderly. Ann Pharmacother 2001;35:1004-9.

5. Kennerfalk A, Ruigómez A, Wallander MA, et al. Geriatric drug therapy and healthcare utilization in the United Kingdom. Ann Pharmacother 2002;36:797-803.

6. Gnjidic D, Hilmer SN, Blyth FM, et al. Polypharmacy cut off and outcomes: five or more medicines were used to identify communitydwelling older men at risk of different adverse outcomes. J Clin Epidemiol 2012;65:989-95.

7. Fialová D, Topinková E, Gambassi G, et al. Potentially inappropriate medication use among elderly home care patients in Europe. JAMA 2005;293:1348-58.

8. Hovstadius B, Hovstadius K, Astrand B, Petersson G. Increasing polypharmacy - an individual-based study of the Swedish population 2005-2008. BMC Clin Pharmacol 2010;2:10-6. 
9. Rome: ItalianDrugs Agency Osservatorio Nazionale sull'impiego dei Medicinali. L'uso dei farmaci in Italia. Rapporto Nazionale Anno 2012. Sept 2013.

10. Hajjar RE, Cafiero AC, Hanlon JT. Polypharmacy in elderly patients. Am J Geriatr Pharmacother 2007;5:345-51.

11. Jyrkkä J, Enlund H, Lavikainen P, et al. Association of polypharmacy with nutritional status, functional ability and cognitive capacity over a three-year period in an elderly population. Pharmacoepidemiol Drug Saf 2011;20:514-22.

12. O'Connor MN, Gallegher P, Byrne S, O'Mahony D. Adverse drug reactions in older patients during hospitalisation: are they predictable? Age Ageing 2012;41:771-6.

13. Maher RL, Hanlon JT, Hajjar ER. Clinical consequences of polypharmacy in the elderly. Expert Opin Drug Saf 2014;13;57-65.

14. The American Geriatrics Society 2012 Beers Criteria Update Expert Panel. American Geriatrics Society Updated Beers criteria for potentially inappropriate medication use in older adults. J Am Geriatr Soc 2012;60:616-31.

15. Onder G, Bonassi S, Abbatecola AM, et al. High prevalence of poor quality drug prescribing in older individuals: a nationwide report from the Italian Medicines Agency (AIFA). J Gerontol A BiolSci Med Sci 2014;69:430-7.

16. Sergi G, De Rui M, Sarti S, Manzato E. Polypharmacy in the elderly: can comprehensive geriatric assessment reduce inappropriate medication use? Drugs Aging 2011;28:509-18.

17. Jyrkkä J, Enlund H, Korhonen MJ, et al. Patterns of drug use and factors associated with polypharmacy and excessive polypharmacy in elderly persons: results of the Kuopio 75+ study: a cross-sectional analysis. Drugs Aging 2009;26:493-503.

18. San-José A, Agustí A, Vidal X, et al. Inappropriate prescribing to the oldest old patients admitted to hospital: prevalence, most frequently used medicines, and associated factors. BMC Geriatr 2015;15:42.

19. Tosato M, Landi F, Martone AM, et al. Potentially inappropriate drug use among hospitalised older adults: results from the CRIME study. Age Ageing 2014;43:767-73.

20. Magalhães GF, Santos GB, Rosa MB, Noblat Lde A. Medication reconciliation in patients hospitalized in a cardiology unit. PLoS One 2014;9:e115491

21. Curatolo N, Gutermann L, Devaquet N, et al. Reducing medication errors at admission: 3 cycles to implement, improve and sustain medication reconciliation. Int J Clin Pharm 2015;37:113-20.

22. Lam MP, Cheung BM. The use of STOPP/START criteria as a screening tool for assessing the appropriateness of medications in the elderly population. Expert Rev Clin Pharmacol 2012;5:187-97.

23. Gallagher P, 0'Mahony D. STOPP (Screening Tool of Older Persons' Potentially Inappropriate Prescriptions): application to acutely ill elderly patients and comparison with Beers' criteria. Age Ageing 2008;37:673-9. 